\title{
Art et nation en Grande-Bretagne : contexte et histoire d'un lien privilégié
}

Art and the Nation in Britain: the Context and History of their Privileged

Connection

\section{Michel Baridon et Frédéric Ogee}

\section{OpenEdition}

\section{Journals}

Édition électronique

URL : http://journals.openedition.org/rfcb/1627

DOI : $10.4000 /$ rfcb. 1627

ISSN : 2429-4373

Éditeur

CRECIB - Centre de recherche et d'études en civilisation britannique

Édition imprimée

Date de publication : 1 janvier 2006

ISSN : 0248-9015

\section{Référence électronique}

Michel Baridon et Frédéric Ogee, «Art et nation en Grande-Bretagne : contexte et histoire d'un lien privilégié », Revue Française de Civilisation Britannique [En ligne], XIII-4 | 2006, mis en ligne le 01 janvier 2006, consulté le 01 mai 2019. URL : http://journals.openedition.org/rfcb/1627 ; DOI : 10.4000/ rfcb. 1627

Ce document a été généré automatiquement le 1 mai 2019.

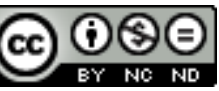

Revue française de civilisation britannique est mis à disposition selon les termes de la licence Creative Commons Attribution - Pas d'Utilisation Commerciale - Pas de Modification 4.0 International. 


\title{
Art et nation en Grande-Bretagne : contexte et histoire d'un lien privilégié
}

\author{
Art and the Nation in Britain: the Context and History of their Privileged \\ Connection
}

Michel Baridon et Frédéric Ogee

1 Il est courant de lire et d'entendre dire que le XVIII ${ }^{e}$ siècle a été le siècle de la prépondérance anglaise. Ces mots servent de titre au volume XI (1715-1763) de la très respectable collection Peuples et Civilisations publiée par les Presses Universitaires de France à la fin des années 1940. La chose a de quoi surprendre: comment un pays qui comptait moins de 6 millions d'habitants en 1700 a-t-il pu devenir plus puissant que la France (20 millions d'habitants au moins) ou que de vastes empires comme ceux de l'Espagne et de l'Autriche? Répondre à cette question, c'est comprendre pourquoi l'Angleterre a suscité beaucoup de curiosité parmi les philosophes des Lumières (Voltaire, Montesquieu, Rousseau s'y sont rendus) et pourquoi les Anglais du XVIII ${ }^{e}$ siècle ont pu avoir le sentiment de constituer une communauté vraiment exceptionnelle.

2 Comme toujours en histoire, il faut remonter un peu en amont d'une période pour la comprendre en profondeur. En 1660, l'Angleterre est sortie d'une longue guerre civile entre les puritains et les royalistes. Les Stuart ont retrouvé le trône d'Angleterre mais la paix civile est demeurée précaire, parce que le parti royaliste s'est appuyé sur les puissances catholiques, ce qui ne correspondait pas aux vœux d'une population majoritairement protestante. Tout majoritaires qu'ils fussent, les protestants n'en étaient pas moins divisés, une grande partie d'entre eux appartenant à l'église réformée nationale, tandis qu'une minorité très active restait fidèle, elle, aux idéaux démocratiques des puritains. Ces dissenters, ces "non-conformistes" ainsi nommés parce qu'ils ne se conformaient pas au culte anglican, se recrutaient particulièrement dans les villes commerçantes et avaient une influence certaine dans les milieux intellectuels. Au cours des années 1680, le conflit s'est aggravé entre, d'une part, la cour et le roi, et d'autre part 
les patriots du parti country (voir l'article Patriot dans l'Oxford English Dictionary). La contestation de l'absolutisme accréditait donc l'idée que la souveraineté résidait dans la nation elle-même.

3 Cette situation s'est prolongée jusqu'en 1688, date à laquelle le roi Jacques II, qui s'était converti au catholicisme, fut chassé du pays par un coup de force militaire. Le stathouder de Hollande, Guillaume d'Orange, protestant bon teint, entra à Londres sans coup férir à la tête d'une armée débarquée sur la côte sud. Jacques II, lâché par le haut personnel politique, s'enfuit en France. Cet événement a été appelé Glorious Revolution parce que, sous ses apparences pacifiques, il fut porteur de transformations importantes qui tendaient toutes à réduire les prérogatives royales et à accroître celles du Parlement et cela, à plusieurs niveaux.

4 Sur le plan dynastique d'abord: Guillaume d'Orange devint Guillaume III roi d'Angleterre mais conjointement avec sa femme Marie, qui détenait un semblant de légitimité en tant que fille du roi déchu. Il n'en restait pas moins que les nouveaux monarques avaient rompu le fil dynastique qui, selon la théorie de l'absolutisme de droit divin, établissait par filiation le caractère sacré du pouvoir royal. La monarchie se présentait désormais comme une monarchie limitée; elle acceptait que son pouvoir, notamment en matière budgétaire, soit contrôlé par le Parlement.

5 Sur le plan des institutions ensuite: le pouvoir suprême résidait désormais dans une entité appelée 'the King in Parliament' ou, selon la formule mille fois répétée, 'King, Lords and Commons'. Conséquence de la victoire des patriots sur les royalists, le monarque ne pouvait plus gouverner sans convoquer le Parlement qui était composé de deux chambres. À la chambre des Lords siégeaient les représentants de la haute aristocratie, 220 familles puissantes par leur ancienneté, par leurs terres, et par le rôle historique qu'elles avaient joué en contestant au besoin l'autorité du roi. Les Lords, c'était un peu la voix de l'histoire et des lieux de mémoire, deux choses qui comptent dans la formation d'une conscience nationale. Les Communes, elles, étaient élues mais selon un système médiéval qui privilégiait la propriété foncière et les campagnes au détriment des villes ; elles ne représentaient que 400000 électeurs mais les grandes questions de politique générale y donnaient matière à des débats dont les journaux se faisaient l'écho. Elles participaient de ce fait à la formation d'une opinion publique d'autant plus vivante que la Glorious Revolution avait supprimé la censure.

6 Sur le plan économique aussi : dans la mesure où le budget faisait l'objet de discussions connues de l'opinion, les affaires économiques devenaient d'intérêt public : instrument décisif de cette politique, la Banque d'Angleterre, créée en 1694 - la Banque de France ne le sera qu'en 1801 -, prit au cours du XVIII ${ }^{\mathrm{e}}$ siècle une importance remarquable. Rien ne nourrit mieux le sentiment national que la conscience d'appartenir à une entité économique entretenant des relations commerciales et financières avec d'autres pays, rivaux ou alliés.

7 Sur le plan politique, enfin: depuis la Réforme, le développement des puissances protestantes autour de la Baltique et de la mer du Nord avait été cause de conflits avec les grandes puissances maritimes catholiques du sud de l'Europe, l'Espagne et la France. L'Atlantique était le théâtre de leurs rivalités. Louis XIV ne pouvant tolérer l'arrivée d'un Hollandais sur le trône d'Angleterre, Guillaume III devint une sorte de champion du protestantisme européen. Les guerres qui en résultèrent furent marquées par des victoires anglaises qui tenaient en échec Louis XIV, présenté comme le Grand Satan, dont le joug maintenait les Français dans la misère. D'où le slogan 'Popery and wooden shoes', qui 
cimentait la cohésion nationale dans la mesure où les protestants de toutes nuances s'accordaient pour protéger le pays de la misère et du papisme.

On peut ainsi voir émerger les lignes d'un large consensus organisé autour de ce que l'on pourrait appeler les acquis de la Glorious Revolution: la paix religieuse, l'espoir de ne plus jamais connaitre de guerre civile ; une extension des libertés puisque, en vertu de l'habeas corpus confirmé solennellement en 1688 , nul ne pouvait être emprisonné sans qu'un tribunal ait statué ; des facilités données à tous pour produire et commercer du fait de la suppression des monopoles de la couronne. La conscience de ces acquis engendra dans l'opinion des valeurs négatives et des valeurs positives. Valeurs négatives : l'absolutisme à la française, toujours dangereux parce qu'il disposait d'alliés potentiels (les Irlandais catholiques, les nostalgiques des Stuart en Angleterre même et en Écosse). Valeurs positives: la réunification des îles Britanniques en 1707 (Union avec l'Écosse), le développement de la puissance économique par la conquête de marchés coloniaux, Londres devenant " a new Rome in the West ", commerçante et civilisatrice.

9 Toutes ces idées circulaient dans le corps social. Certes, elles ne faisaient pas l'unanimité : des conflits existaient entre non-conformistes et anglicans, eux mêmes divisés en High Church et Low Church selon leur conception de l'orthodoxie; les milieux d'affaires ( moneyed interest) et les propriétaires terriens (landed interest) étaient en désaccord sur les problèmes de finance et de politique extérieure; dans la seconde moitié du siècle, la révolution industrielle et la croissance de la population causèrent des crises graves. Mais il n'empêche. Entre la Glorious Revolution de 1688 et le Reform Bill de 1832 la stabilité sociale s'est maintenue grâce à un consensus national fondé, selon J.H. Plumb, sur 'a balancing of the tensions'.

10 Le patriotisme anglais n'est pas né avec la Glorieuse révolution; sous les Tudor on en trouve des expressions nombreuses et célèbres, chez Shakespeare notamment. Néanmoins des faits révélateurs se sont produits au XVIII siècle: après l'acte d'union avec l'Écosse (1707), la croix de St André apparaît dans l'Union Jack qui devient le drapeau de Britannia et des Britons. Dans les années 1710, Arbuthnot crée le personnage de John Bull, franc, tenace mais agressif si l'on touche à sa liberté; ce personnage tient encore, dans la caricature, le même rôle que l'Oncle Sam aux États-Unis et que Marianne en France. En 1740, on joue pour la première fois l'hymne patriotique Rule Britannia, Britannia rule the Waves, musique de Thomas Arne, paroles de James Thomson. Cet air souvent entendu encore à la fin des Promenade Concerts exalte le destin impérial de la GrandeBretagne maîtresse des mers; il affirme 'Britons never shall be slaves', ce qui revient à dire : " conquérons un empire et soyons libres chez nous ». Cinq ans plus tard, l'orchestre de Drury Lane joue God Save the King pour saluer l'entrée de Georges II dans ce théâtre; le pays vit alors une crise majeure, celle de la tentative avortée de l'héritier des Stuart déchus de descendre sur Londres depuis l'Écosse. L'habitude est vite prise de jouer ce morceau chaque fois que le roi apparait en public ; il acquiert le statut d'hymne national pendant une autre crise majeure, celle des guerres napoléoniennes, et devient ainsi l'expression musicale du sentiment national. Et ce sentiment se retrouve dans bien d'autres manifestations de la vie culturelle. C'est bien lui en effet qui permet de comprendre la rapide apparition, au cours du XVIII ${ }^{e}$ siècle, d'une "École anglaise de peinture ", et c'est lui, surtout, qui motive les conditions dans lesquelles elle s'est définie. Prise en charge par les artistes eux-mêmes dès le début du siècle, mais aussi attisée par le dynamisme du marché de l'art à Londres, la question du rapport entre la nation et sa production et «consommation» artistiques fournit à la Grande-Bretagne l'occasion 
d'exprimer un point de vue tout à fait original et novateur qui eut pour effet, non seulement de doter le pays d'une institution à la hauteur de ses ambitions artistiques, la Royal Academy of Arts, mais aussi de faire entendre une voix résolument anglaise en matière de production artistique autant que de débats esthétiques.

S'il y avait depuis longtemps de la peinture en Angleterre, il n'y avait pas pour autant de peinture anglaise (distinction qui mériterait d'ailleurs d'être davantage problématisée). Jusqu'au début du XVIII ${ }^{e}$ siècle, la production picturale y fut l'œuvre quasi exclusive d'artistes étrangers souvent prestigieux, attirés à grands frais par les monarques et leurs cours pour témoigner de leur splendeur. Henri VIII avait choisi l'Allemand Hans Holbein, le plus grand portraitiste de l'humanisme européen, pour imposer à travers l'Europe son image de monarque puissant - et protestant - et ainsi inaugurer une iconographie royale officielle et anglaise (quel livre d'histoire ne contient pas ces portraits?) ${ }^{1}$. Si sa fille cadette, qui semble avoir eu peu de goût pour l'art (trop catholique?), confia ses représentations à des miniaturistes remarquables mais dont l'art confine davantage à la joaillerie (Nicholas Hilliard, Isaac Oliver), les monarques Stuart qui lui succèdent firent à nouveau appel à des artistes cosmopolites de renom pour intégrer leur image et leur cour dans un paysage européen. Jacques $\mathrm{I}^{\mathrm{er}}$, puis Charles $\mathrm{I}^{\mathrm{er}}$, ne parvinrent pas à retenir à Londres le très catholique diplomate Rubens après que celui-ci eut néanmoins réalisé (mais après son retour en Flandre...) le plafond du Banqueting Hall de Whitehall (1629-34), mais son plus prestigieux disciple Anthony Van Dyck passa les années 1630 à Londres et imposa en Angleterre un style de portrait en pied dont l'influence profonde se fit sentir chez les peintres britanniques jusqu'au $\mathrm{XX}^{\mathrm{e}}$ siècle.

Après l'épisode Cromwell et son iconoclasme forcené, Charles II et sa cour, restaurée mais moribonde, élevèrent le Hollando-Allemand Peter Lely, déjà actif sous Charles $\mathrm{I}^{\mathrm{er}}$ et Cromwell, au rang de 'Principal Painter in Ordinary' et multiplièrent les commandes de portraits où, sans craindre le paradoxe, l'artiste représenta cette « restauration » dans une atmosphère assez lugubre de fin de règne ${ }^{2}$. Enfin, après la chute de Jacques II et l'avènement de Guillaume d'Orange puis de la reine Anne et enfin des Hanovre, c'est l'Allemand Godfrey Kneller, originaire de Lübeck mais actif en Angleterre dès 1676, qui présida aux destinées de l'art en Angleterre, concurrencé seulement par le Suédois Michael Dahl, et assura une forme de trait d'union entre les deux époques.

Car c'est bien sous son impulsion, et peut-être parce qu'il avait été le "spectateur » privilégié des soubresauts et bouleversements profonds qui affectèrent l'Angleterre pendant toute cette période charnière entre le XVII ${ }^{e}$ et le XVIII ${ }^{e}$ siècle, qu'eut lieu non seulement l'apparition d'un nouveau style plus informel de portrait (le 'Kit-Cat'), mais aussi la fondation d'une première académie de peinture à Londres, la Queen Street Academy , véritable creuset de jeunes talents, majoritairement anglais, qui, comme tous leurs contemporains, ne demandaient qu'à "progresser ». Si cette académie n'eut guère d'influence véritable sur la formation des artistes (il s'agit davantage d'un club, comme il s'en crée tant à la même époque), elle permit le regroupement et accrut la visibilité d'une génération de peintres (quasi exclusivement des portraitistes) dont l'éclosion coïncida avec la disparition d'un mécénat de cour vertical et élitiste au profit d'une demande abondante et bien dotée émanant de ces «classes moyennes" issues de la Glorious Revolution qui, dans tous les sens du terme, ambitionnaient de représenter la nouvelle nation britannique.

On sous-estime parfois, dans un pays privé d'académie de peinture, le rôle formateur sans parler du considérable rendement quantitatif - des ateliers dirigés par les grands 
peintres étrangers présents sur le sol anglais jusqu'à l'avènement des Hanovre. De Holbein à Kneller, tout étrangers qu'ils aient pu être, il y a bien eu grâce à ces artistes remarquables une véritable «invention de traditions » outre-Manche, et l'affleurement progressif d'un «style anglais", dont la dette envers les influences continentales mériterait une analyse plus approfondie. Quoi qu'il en soit, la génération des portraitistes qui rencontre son public dans les premières décennies du XVIII siècle (c'est l'âge d'or de la conversation piece) souffre néanmoins d'un manque de reconnaissance statutaire et sociale (on les considère comme des artisans, leurs œuvres font partie des meubles). De Shaftesbury (en 1711) à Horace Walpole (dans ses Anecdotes of English Painting de 1762-65, au titre significatif), l'élite du pays considérait ce début de siècle comme une période noire pour l'art en Angleterre ${ }^{3}$.

Mais, on l'a dit plus haut, la nation britannique naissante est elle en pleine effervescence, et le besoin d'affirmer sa grandeur et de se débarrasser de ses complexes d'infériorité ( 'Britons never shall be slaves') s'exprime à tous les niveaux, y compris celui des arts. Éprouvant un vif désir de reconnaissance, la Grande-Bretagne est alors en quête de nouvelles formes de représentation d'elle-même qui lui permettront à la fois de se connaître (c'est l'âge d'or des manuels de savoir-vivre et autres conduct-books) et de se faire connaître. Dès la deuxième décennie vont commencer à apparaitre, plus ou moins confusément, diverses formes nouvelles d'expression artistique, en phase avec les idées «modernes » et destinées à prendre en charge ce besoin de reconnaissance. Ce seront les premières longues fictions « réalistes» en prose (celles d'un Defoe, surtout), que l'on n'appelle pas encore des romans, mais aussi les premières esquisses d'une dégéométrisation du jardin à la française - jusqu'alors le modèle dominant dans toute l'Europe - au profit d'un espace plus naturel et expérimental (landscape garden) qui trouve son inspiration et son esthétique du côté de la peinture et non plus de l'architecture ( «ut pictura hortus »). On comprend bien, dans ce contexte, la pression qui a pu s'exercer alors sur le monde de l'art et l'ensemble de ses acteurs pour faire émerger également une véritable "École anglaise de peinture" digne de ce nom et destinée à égaler ses homologues européennes, et ainsi mettre fin à une anomalie, une carence, constatée à l'envi et non sans malice par nombre de visiteurs étrangers lors de leurs voyages outreManche.

es années 1740 - decennium mirabile - constituent à coup sûr le moment décisif de la rencontre entre les nouvelles formes de représentation artistique et les idées-forces de la nation. Qu'on en juge: en fiction, c'est la formidable série entrecroisée des romans " modernes » de Richardson et Fielding; dans les jardins paysagers, après la vibrante épître de Pope à Burlington (Moral Essay IV, 1731, 'Of False Taste' : 'In all, let Nature never be forgot... Consult the genius of the place in all'), ce sont les grandes heures de Stourhead et de Stowe et le passage de témoin entre les audaces formelles de William Kent (le parcours sinueux, le ha-ha) et le paysagisme sensible de Brown; en peinture, enfin, c'est l'apogée du génie de Hogarth (Marriage à-la-Mode, The Graham Children, Self-Portrait with Pug), mais aussi les débuts remarquables de Gainsborough (Cornard Wood, Mr. and Mrs. Andrews) de Reynolds et de Allan Ramsay (qui s'installe à Londres en 1739).

17 Afin de rehausser et d'affirmer le statut des peintres anglais et de préciser leur utilité au sein de la nation, il convenait clairement de doter celle-ci d'une institution à la charge symbolique forte, qui assoie leur présence au sein de la cité, tout en préservant la sacrosainte liberté des artistes, qu'une académie des beaux-arts sur le modèle français ne manquerait pas de mettre à mal. Alors que le pays s'était par ailleurs doté rapidement 
d'une panoplie d'institutions et d'instruments « modernes » à la hauteur de ses ambitions (le système potentiellement contractuel et représentatif de la monarchie parlementaire en politique; la Banque d'Angleterre - et donc le principe de la dette nationale -, les grandes « compagnies » commerciales et l'introduction du papier-monnaie en économie ; la Royal Society en science), il apparaissait de plus en plus nécessaire de parachever ce vaste projet de civilisation par un geste fort en direction des arts. Le marché de l'art était florissant et Londres avait vite remplacé Amsterdam comme plaque tournante du commerce des œuvres (James Christie fonde sa célèbre salle des ventes en 1766). Des expositions de peinture anglaise commençaient à apparaître et rencontraient un grand succès (dans les pleasure gardens de Vauxhall et Ranelagh, et, à l'initiative de Hogarth, au Foundling Hospital fondé par Thomas Coram en 1739, où tous les futurs grands noms de la peinture anglaise exposeront), et un discours britannique sur l'art (Shaftesbury, Addison, Jonathan Richardson, Hutcheson, Hogarth, Gerard, Burke, Kames, Reynolds) se construisit tout au long du siècle et contribua de manière décisive à l'émergence de l'esthétique en tant que discours philosophique autonome.

Les artistes anglais tentèrent bien de s'organiser entre eux, mais divergences de vues et rivalités, souvent dues à leur origine sociale différente, entraînèrent la scission de la Queen Street Academy et la formation d'une structure concurrente, la St Martin's Lane Academy, animée par Hogarth dans un esprit résolument démocratique et anti-élitiste. De leur côté, faisant écho aux écrits de Shaftesbury, les élites, notamment la Society of Dilettanti (fondée en 1734, elle regroupait surtout des aristocrates rentrés de leur Grand Tour abreuvés d'art ancien), tentèrent de prendre le contrôle des destinées artistiques de la nation, au nom d'un prétendu « intérêt supérieur » (civic humanism), afin d'y imposer leurs normes en matière de goût et de sujets, mais leur démarche rencontra l'hostilité des artistes, qui n'entendaient pas se faire dicter leur travail et ainsi être maintenus dans un statut de simples exécutants.

Une nouvelle tentative de fédération et d'organisation des artistes eut lieu au début des années 1760, avec la création de la Society of Artists of Great-Britain, puis de la Free Society of Artists, dissidente de l'autre, mais à nouveau les dissensions internes en minèrent le projet. Seule une impulsion venue "d'en haut ", un signe fort au nom de la nation, était susceptible de faire passer ces querelles au second plan (elles ne cesseront jamais). Emmené par l'architecte William Chambers, qui avait l'oreille du monarque, un groupe d'artistes (parmi lesquels figuraient Benjamin West, Nathaniel Dance, Richard Wilson, et les deux futures académiciennes Mary Moser et Angelica Kauffman) parvint à convaincre Georges III d'accorder son parrainage à une grande institution de prestige, la Royal Academy of Arts, qui put ainsi être enfin fondée, à Londres, le 10 décembre 1768. En ouverture au premier Discours qu'il y prononça à peine trois semaines plus tard en tant que Premier président (élu à l'unanimité par ses pairs), le peintre Joshua Reynolds tint à souligner la nécessité ('must') de reconnaître l'importance des enjeux nationaux d'une telle entreprise: 'An Academy, in which the Polite Arts may be regularly cultivated, is at last opened among us by Royal Munificence. This must appear an event in the highest degree interesting, not only to the Artists, but to the whole nation'. (Joshua Reynolds, Discourse I, January 2,1769). Précision importante : cette institution était autonome, auto-financée (grâce aux expositions annuelles) et entièrement administrée par les artistes eux-mêmes. Emblème d'une volonté nationale, elle était indépendante du pouvoir. Et dès 1780 , l'Académie emménageait dans des locaux également de prestige, Somerset House, dessinés par Chambers entre le Strand et la Tamise, où, de façon aussi symbolique que 
significative, elle cohabita avec l'administration des douanes (source essentielle de la prospérité britannique) et celle de la Royal Society, à l'origine du nouveau regard que les Anglais portaient sur le monde.

Les premières années du règne de Georges III (sur le trône depuis 1760) semblaient particulièrement propices à la formation, enfin, d'une véritable association d'artistes, autour d'un vrai projet de dimension nationale ${ }^{4}$. C'est en effet dans ces années-là qu'apparut de manière explicite un "sentiment national», enfin dégagé de la problématique jacobite, confirmant le souhait d'un engagement collectif et conquérant sur la scène du monde, et affichant la certitude de son existence et de sa pérennité au point de vouloir se doter d'institutions et de bâtiments qui en concrétisent et en affichent la grandeur.

21 Le pays était alors très prospère et les soucis domestiques avaient cessé de mobiliser les énergies. Depuis une dizaine d'années régnaient paix et stabilité. Une véritable culture du loisir, et donc une vraie demande artistique, semblaient désormais possibles, grâce notamment à l'afflux constant de richesses permis par le commerce. L'artisanat domestique était déjà d'une qualité exceptionnelle, mais, on l'a dit, la production nationale de peinture lui restait assimilée et ressortissait encore de l'ameublement.

La grande ambivalence du débat, au cœur du projet complexe de fondation d'une académie britannique, résidait dans la nécessité peu à peu mise au jour de concilier les hautes ambitions artistiques de la nation et les exigences d'un marché de l'art qui, pour toutes les raisons décrites ci-dessus, était également apparu dans le Londres du XVIII ${ }^{e}$ siècle. En effet, l'extraordinaire prospérité des Britanniques s'était traduite, on comprend bien pourquoi, par un véritable engouement pour l'acquisition d'œuvres d'art destinées, comme pour les Hollandais au siècle précédent, à donner des gages de respectabilité intellectuelle et de responsabilité sociale, au sein d'un univers où la réussite matérielle, voire l'appât du gain, devaient être en quelque sorte «blanchis » par un intérêt marqué pour les œuvres de l'esprit. Si d'un côté on cherchait sans vergogne à faire de bonnes affaires, y compris dans l'acquisition privée d'œuvres d'art, il fallait pouvoir démontrer que cette démarche s'inscrivait aussi dans un dessein public de promotion d'une grande culture britannique, au service de l'idéal humaniste de la nation.

23 À partir des années 1750 , c'est un véritable public au sens moderne du mot qui était apparu, grâce à la multiplication des expositions et des possibilités de voir et d'acquérir des œuvres d'art. Cela s'accompagna de la parution de journaux et d'ouvrages donnant des conseils sur la bonne façon d'évaluer et de regarder celles-ci, qui contribuèrent à transformer une pratique jusqu'alors très nettement restreinte à une élite en un véritable phénomène social. L'élargissement et l'enrichissement des nouvelles classes moyennes provoqua ainsi très rapidement une évolution radicale du rapport social à l'art et aux artistes, qui eut pour effet de diversifier la demande et de mettre sur la place publique un débat et une activité qui étaient restés confinés dans des cercles très fermés. Au-delà des effets de mode qui pouvaient affecter ce public et de la relative superficialité de son intérêt pour les questions d'esthétique, cette transformation fut déterminante pour modifier les termes du débat et le sortir de l'impasse dans laquelle il semblait être. Elle fut cruciale pour la naissance de la Royal Academy. Entre une élite exigeant (et achetant) du grand art et des peintres anglais incapables d'en produire en ces termes, l'apparition d'un segment intermédiaire d'acheteurs de plus en plus nombreux, aux fortunes diverses, et dont les valeurs et les priorités étaient davantage en phase avec celles des artistes alors en activité créait de facto un lien entre les deux extrêmes et permit l'émergence d'une 
sorte de continuum de l'offre et de la demande, du plus modeste au plus ambitieux, qu'une institution comme la Royal Academy, administrée par les artistes eux-mêmes, allait se charger d'organiser afin de tirer tout le monde vers le haut (tant les acheteurs que les artistes) et ainsi promouvoir les idéaux artistiques de la nation en ménageant les intérêts des uns et des autres.

Cet afflux d'un public plus marchand que connaisseur ne fut pas sans poser de sérieux problèmes à la nouvelle académie lors des expositions annuelles qu'elle organisa dès l'origine pour faire connaître les œuvres des artistes anglais. Mais il est important de comprendre que la principale raison d'être de l'Academy à la date où elle fut fondée, et l'énorme engouement qu'elle suscita immédiatement, s'expliquent avant tout par la volonté de ses principaux instigateurs de lui faire occuper une position charnière entre artistes vivants et grand public, entre les idéaux de l'art et les pressions du marché. Conçue dans le but de promouvoir les arts graphiques et plastiques (essentiellement la peinture à l'huile, la sculpture et l'architecture), elle chercha constamment, sans toujours l'atteindre, le juste équilibre entre les nostalgies élitistes de l'aristocratie et les impatiences marchandes des nouvelles classes moyennes (dont étaient issus la plupart des artistes).

C'est ce qui permet de comprendre l'organisation et le partage de ses activités, entre formation théorique et pratique d'une grande exigence (pour élever le niveau général des artistes) d'un côté, et grande exposition annuelle à forte visibilité sociale (pour permettre à ces artistes de se faire connaitre - et donc de vivre de leur art - et au public d'éduquer son goût au contact d'un art anglais sélectionné et ambitieux) de l'autre. Au cœur de sa conception se trouvait un vaste projet éducatif, au service des ambitions de la nation et à l'écoute de ses ambiguïtés.

Il est difficile de mesurer l'importance véritable qu'a pu avoir cet adoubement institutionnel dans l'émergence d'une école nationale de peinture (quel que soit le sens que l'on puisse donner à cette notion...) en Grande-Bretagne. On ne peut que constater le remarquable succès international rencontré par les artistes britanniques à la fin du XVIII ${ }^{e}$ et au début du XIX ${ }^{e}$ siècle. En quelques décennies, ce sont les œuvres de Reynolds, de Gainsborough, de Raeburn, puis de Constable et de Turner qui constitueront l'un des grands moments de la créativité artistique outre-Manche. Mais sans parler des cas de Blake ou de Romney ('British art's forgotten genius' comme le sous-titrait l'exposition de 2002 à la Walker Art Gallery de Liverpool), qui refusèrent tout lien avec l'Academy, on doit aussi admettre que Reynolds et Gainsborough étaient déjà célèbres en 1768, et que les rapports de Constable et de Turner à l'institution furent pour le moins ambivalents.

Peut-être devrait-on, en guise de conclusion, évoquer la question récurrente, lancinante et parfois naïvement posée de 'the Englishness of English art'. Quels rapports profonds cette peinture entretient-elle avec l'esprit (ethos/psyche) britannique, quelles «relations spéciales » cet art a-t-il établies avec cette nation ? Contentons-nous ici de considérations historiques : on le rappelait au début de cet essai, depuis la fin du XVII (peut-être même depuis Bacon) et tout au long du XVIII ${ }^{e}$ siècle, la Grande-Bretagne a apporté une contribution décisive, et souvent sous-estimée, à l'Europe des Lumières. Faisant de l'idée de liberté l'emblème d'une nation conquérante, et sans oublier les relents nauséabonds des workhouses, de l'esclavagisme et bientôt de l'impérialisme «civilisateur » qui ont pu émaner de cette conquête tonitruante du monde, les Britanniques ont tenté de façonner un modèle social au sein duquel chaque individu pouvait effectuer son propre progress. $\mathrm{Ce}$ principe du "parcours», profondément inspiré par les nouvelles épistémologies 
lockienne et newtonienne diffusées par une presse dynamique et éclairée, a participé de ce fameux désenchantement du monde, selon la formule célèbre de Max Weber, qui caractérise la période. Permettant l'accès direct, non médiatisé, d'un plus grand nombre (à défaut du plus grand nombre) aux " plaisirs de l'imagination ", il a notamment permis l'instauration d'un rapport nouveau et «moderne » aux œuvres d'art, d'une manière qui ne s'est jamais vraiment démentie depuis. L'éclosion d'une école anglaise de peinture s'est ainsi faite à la faveur d'un rapprochement inédit entre art et nation, et si l'on examine ses genres de prédilection - le portrait et le paysage - on constate qu'ils sont la traduction parfaite dans le domaine artistique des postulats empiristes qui prônaient la découverte et l'observation attentive des phénomènes de la Nature, y compris la nature humaine. En proposant à leurs contemporains, qui les y encourageaient, des représentations d'euxmêmes - au naturel ou en situation - et de leur pays (le lieu a du génie, avait dit Pope, et ce sera tout le propos du picturesque), les artistes anglais ont offert à leur nation un répertoire d'images, certes souvent flatteuses, mais résolument « naturelles », dans lequel ils ont pu puiser une identité (inwardly) et une publicité (outwardly).

Et ceci vaut encore aujourd'hui. Lors d'une enquête menée à l'été 2005, la BBC a demandé à ses auditeurs de choisir l'œuvre d'art la plus importante exposée selon eux dans les collections britanniques. Dans la liste finale de dix tableaux, où ne figure aucune œuvre de Holbein, Raphaël, Van Dyck, Rubens ou Rembrandt, on ne dénombre pas moins de six toiles d'artistes britanniques (Hogarth, Raeburn, Constable, Turner, Ford Madox Brown et Hockney). Comme l'écrit l'historien d'art Richard Dorment:

The paintings that have made the shortlist have in common a strong, powerful central image in which we can recognise something of ourselves, some true emotion. The Last of England [Brown] is about departure, The Fighting Temeraire [Turner] about retirement [...]. Even The Hay Wain [Constable], apparently the most hackneyed of landscapes, has something to say about the idyll of our childhoods and the timeless sensation of an English summer. ${ }^{5}$

Au-delà de toute autre considération esthétique ou politique, ce choix confirme un lien privilégié et vivace entre la nation britannique et son art, empreint d'autant de nostalgie que de fierté, et qui semble assez unique dans l'histoire de la peinture occidentale.

\section{NOTES}

1. Voir là-dessus le tout récent ouvrage de Susan FOISTER, Holbein and England, New Haven \& London: Yale University Press, 2005.

2. Voir sur cette peinture le remarquable catalogue de l'exposition Painted Ladies: Women at the Court of Charles II. Catharine MACLEOD \& Julia MARCIARI ALEXANDER, eds. New Haven \& London: Yale University Press, 2001.

3. Walpole écrit que, dans les premières années du règne de Georges I ${ }^{\text {er }}$ 'the arts were sunk to their lowest ebb in Britain' (Anecdotes, p. 316).

4. Les quelques paragraphes qui suivent reprennent en partie le texte de notre Introduction à Isabelle BAUDINO, Jacques CARRÉ \& Frédéric OGÉE, Art et Nation : la fondation de la Royal Academy of Arts, 1768-1836, Paris : Armand Colin, 2004. 
5. Daily Telegraph, 16 August 2005. Article reproduit sur : http://www.telegraph.co.uk/arts/ main.jhtml?xml=/arts/2005/08/16/babest16.xml\&sSheet=/arts/2005/08/16/ixtop.html

\section{RÉSUMÉS}

Dans l'histoire de l'Europe, le XVIII ${ }^{\mathrm{e}}$ siècle est souvent considéré comme celui de la prépondérance anglaise. La Glorieuse révolution de 1688 avait chassé Jacques II du trône, mettant fin à la monarchie absolue, héréditaire de droit divin, pour la remplacer par une monarchie constitutionnelle, dans laquelle le pouvoir était entre les mains du Parlement. À ce progrès politique s'ajoutèrent liberté religieuse et prospérité économique, donnant naissance à un patriotisme incarné par les figures emblématiques de Britannia, de John Bull en littérature, par l'hymne national du Rule Britannia de Thomson, mis en musique par Thomas Arne. Ce sentiment de fierté nationale se retrouve dans la création d'une "école anglaise de peinture ", grâce à la fondation de la Royal Academy of Arts en 1768. C'est l'aboutissement de toute une évolution qui a marqué les années 1740 - decennium mirabile - par la rencontre entre les idées forces de la nation et les nouvelles formes d'expression artistique : roman, jardin paysager, peinture. Un nouveau public cossu apparaît, amateur des expositions qu'organise la Royal Academy et des œuvres offertes sur le marché de l'art.Quels rapports entre cet art et l'esprit britannique ? C'est tout le problème de « l'anglicité de l'art anglais », du lien entre la nation britannique et ses arts au XVIII ${ }^{\mathrm{e}}$ siècle.

In the history of Europe, the eighteenth century is often considered as the period of British supremacy. The Glorious Revolution (1688) had driven James II from the throne, putting an end to absolute and hereditary monarchy by divine right, replacing it by a constitutional monarchy in which the ultimate power lay in the hands of Parliament. That political progress was completed by religious freedom and economic prosperity, giving birth to a feeling of national pride, embodied by the emblematic figures of Britannia, John Bull in literature, and the national anthem, Rule Britannia, written by James Thomson and set to music by Thomas Arne. This feeling of national pride can be found in the development of an 'English school of painting', with the foundation of the Royal Academy of Arts in 1768. It was the outcome of an evolution that characterised the 1740s - decennium mirabile - by the convergence between the basic ideals of the nation and the new forms of artistic expression: the novel, landscape gardening and painting. A new wealthy public enjoyed the exhibitions organised by the Royal Academy and the works displayed thanks to the art market. What connection is there between this art and the British psyche? The answer to that question raises the issue of 'the Englishness of English art', of the link between the British nation and its art in the eighteenth century.

\section{AUTEURS}

\section{MICHEL BARIDON}

Université de Dijon 
FRÉDÉRIC OGEE

Université de Paris VII 